\title{
5G: Vision and Requirements for Mobile Communication System towards Year 2020
}

\author{
Guangyi Liu and Dajie Jiang \\ China Mobile Research Institute, Beijing 100053, China \\ Correspondence should be addressed to Guangyi Liu; liuguangyi@chinamobile.com
}

Received 8 October 2015; Accepted 2 March 2016

Academic Editor: Juho Lee

Copyright ( 2016 G. Liu and D. Jiang. This is an open access article distributed under the Creative Commons Attribution License, which permits unrestricted use, distribution, and reproduction in any medium, provided the original work is properly cited.

\begin{abstract}
The forecast for future 10 years' traffic demand shows an increase in 1000 scales and more than 100 billion connections of Internet of Things, which imposes a big challenge for future mobile communication technology beyond year 2020. The mobile industry is struggling in the challenges of high capacity demand but low cost for future mobile network when it starts to enable a connected mobile world. 5G is targeted to shed light on these contradictory demands towards year 2020. This paper firstly forecasts the vision of mobile communication's application in the daily life of the society and then figures out the traffic trends and demands for next 10 years from the Mobile Broadband (MBB) service and Internet of Things (IoT) perspective, respectively. The requirements from the specific service and user demands are analyzed, and the specific requirements from typical usage scenarios are calculated by the defined performance indicators. To achieve the target of affordable $5 \mathrm{G}$ service, the requirements from network deployment and operation perspective are also captured. Finally, the capabilities and the efficiency requirements of the 5G system are demonstrated as a flower. To realize the vision of 5G, "information a finger away, everything in touch," $5 \mathrm{G}$ will provide the fiber-like access data rate, "zero" latency user experience, and connecting to more than 100 billion devices and deliver a consistent experience across a variety of scenarios with the improved energy and cost efficiency by over a hundred of times.
\end{abstract}

\section{Introduction}

The first generation of mobile communication system based on analog signal was born in the 1980s, and it helped people get rid of the shackles of telephone line. In the 1990s more efficient second-generation (2G) mobile communication systems based on digital communication occurred, and after that personal mobile communications have had a rapid development on a global scale. After 2000, with the deployment of $3 \mathrm{G}$ systems, people can enjoy faster mobile Internet experience, such as video telephony. When it comes to 2010, deployment of Long Term Evolution (LTE) based 4G commercial network further enhanced the system capacity and user experience. According to the statistics of Global TD-LTE Initiative (GTI), 364 LTE commercial networks have been launched by the third quarter of 2015. The evolution map of mobile communications since 1980s is summarized in Figure 1. With the IMT-Advanced (IMT-A) systems being deployed in the world, the 5th-Generation (5G) mobile communication technologies are emerging into research fields.
In order to drive future development of mobile communication techniques, the METIS (Mobile and Wireless Communications Enablers for the Twenty-Twenty Information Society) project [1] of European Union started research work of 5G at the end of 2012. In China, IMT-2020 promotion group was founded in April of 2013. IMT-2020 promotion group will serve as a platform to promote the 5G study. Its target is to organize domestic forces to actively carry out international cooperation and to jointly promote the international development of 5G. In Korea, Samsung tested and verified the technical feasibility of millimeter wave in the bands of about $28 \mathrm{GHz}$ [2]. Other possible candidate technologies such as massive MIMO [3], novel multiple access [4], and new channel coding [5-7] have attracted more and more interest. The International Telecommunication Union (ITU) has also started its study on the International Mobile Telecommunication system towards 2020 (IMT-2020) since 2013 [8]. Third Generation Partnership Project (3GPP) will start its study and standardization work on IMT-2020 from March 2016 [9]. 


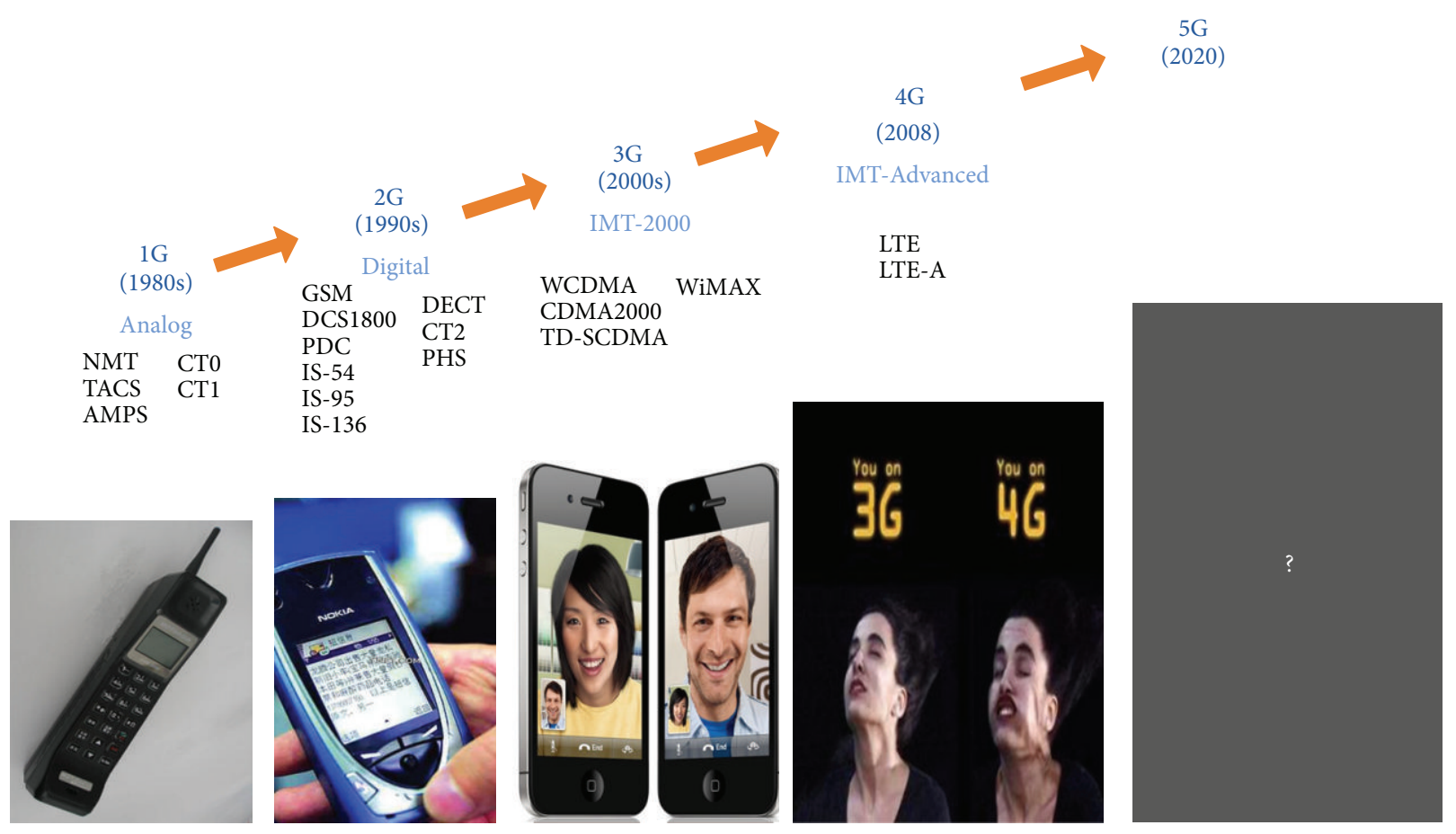

FIGURE 1: Evolution map of mobile communications.

This paper discusses the vision of the mobile communication towards year 2020 first, and the capabilities of 5G system are derived on condition that a sustainable ecosystem of mobile communication system could be built to meet the market demand of year beyond 2020 .

\section{Vision for Mobile Communication towards 2020}

The global deployment of LTE cultivates the mobile users to be used to the mobile data in their daily life tremendously. The video service and social applications, for example, WeChat, Facebook, and Twitter, have changed our life very much with the capabilities of LTE, especially high data rate and low latency. It is believed that the mobile communication will penetrate into every element of future society and create an all-dimensional, user-centered information ecosystem. A fully mobile and connected society is expected in the near future, which will be characterized by a tremendous amount of growth in connectivity, traffic volume, and a much broader range of usage scenarios [10].

Accordingly, the Mobile Broadband (MBB) service and the Internet of Things (IoT) will be the two main drivers in the future development of mobile communications, and they will provide a broad prospect for the next generation mobile communication system (5G), the overall vision of which is depicted in Figure 2.

Mobile Broadband service disrupted the traditional business model of mobile communications, enabling unprecedented user experiences and making a profound impact on every aspect of people's work and life. Looking ahead to year 2020 and beyond, MBB service will promote the continued evolution of the way humans interact and provide users with ultimate experience through more immersive services such as augmented reality, virtual reality, ultra-high-definition (UHD) 3D video, and mobile cloud. The further development of mobile Internet will trigger the growth of mobile traffic by a magnitude of thousands in the future and promote a new wave of upgrades and a revolution in mobile communications technologies and the industry as a whole.

Looking ahead to the year 2020 and beyond, there will be explosive growth in mobile data traffic as shown in Figure 3. It is estimated that the global mobile data traffic will grow by more than 200 times from 2010 to 2020 and by nearly 20,000 times from 2010 to 2030 . In China, the growth factors are projected to be even higher, with mobile data traffic being expected to grow by more than 300 times from 2010 to 2020 and by more than 40,000 times from 2010 to 2030 . For developed cities and hotspots in China, the growth of mobile data traffic will exceed the projected average growth for all of China. For example, from 2010 to 2020 in Shanghai, the mobile data traffic is projected to grow by 600 times. In Beijing and during this same period, it is estimated that hotspot traffic may grow by up to 1,000 times.

The IoT has extended the scope of mobile communications services from interpersonal communications to smart interconnection between things and between people and things, allowing mobile communications technologies to penetrate into broader industries and fields. Looking ahead to the year 2020 and beyond, applications such as mobile health, Internet of Vehicles (IoV), smart home, industrial control, and environmental monitoring will drive the explosive growth of IoT applications, facilitating hundreds of billions of devices to connect to a network creating a true 


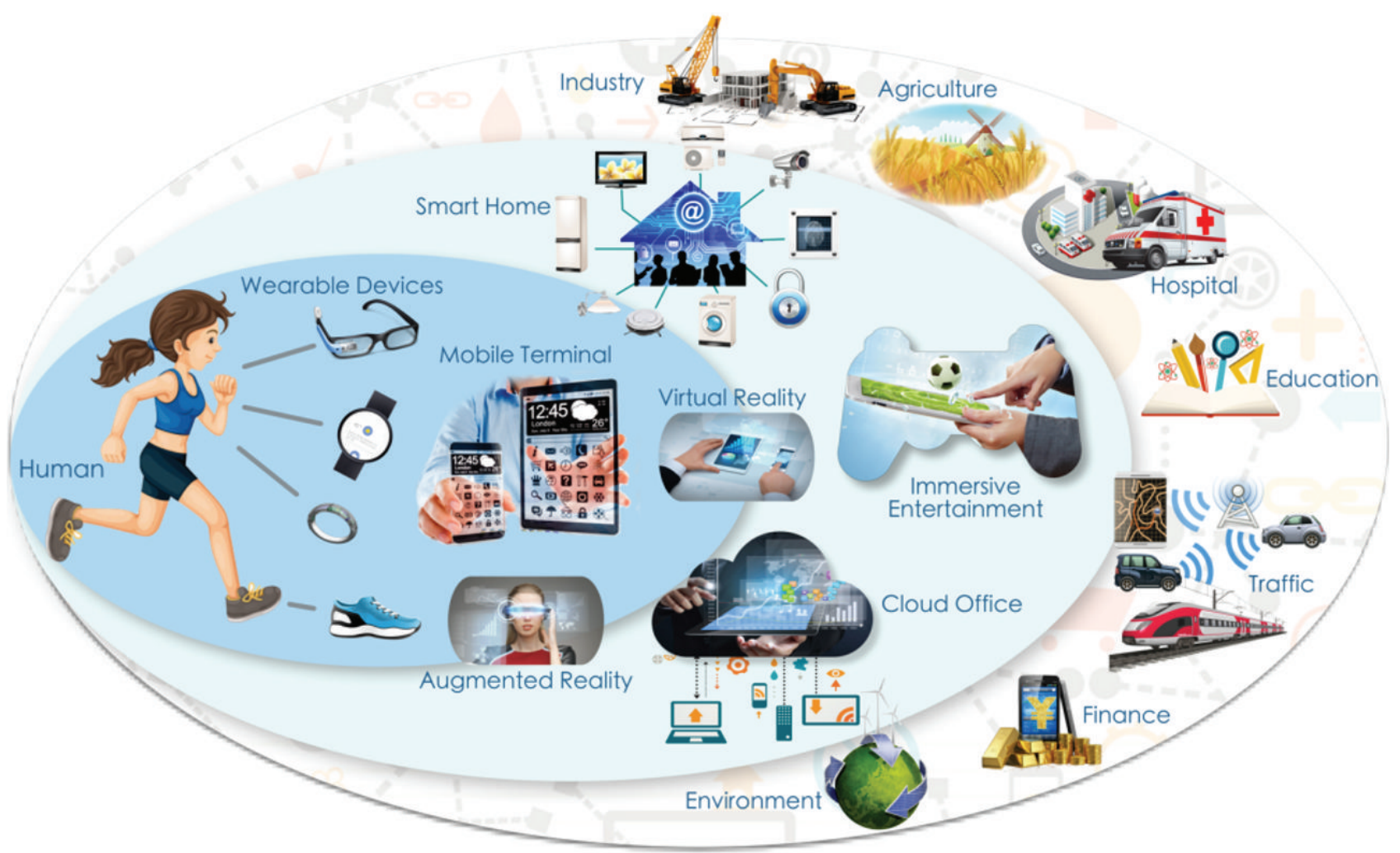

FIGURE 2: Overall visions of 5G [10].

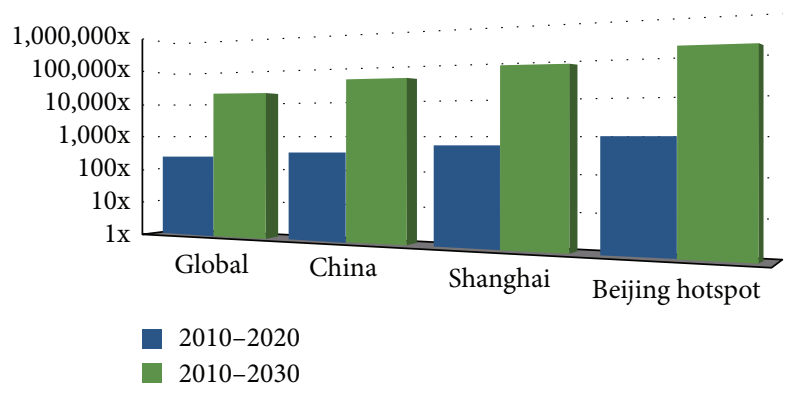

Figure 3: 2010-2030 growth of mobile data traffic [10].

"Internet of Everything." This will give rise to emerging industries of unprecedented scale and instill infinite vitality to mobile communications. Meanwhile, the massive amount of interconnected devices and the diversified IoT services will also pose new challenges to mobile communications.

As illustrated in Figure 4, the total number of devices connected by the global mobile communications network will reach 100 billion in the future. By 2020, it is projected that the number of mobile terminals around the world will surpass 10 billion, of which China will contribute over 2 billion. The number of IoT connections will also expand rapidly, reaching the size of the global population of 7 billion in 2020, of which China will contribute 1.5 billion. By 2030, the number of global IoT connections will reach 100 billion, of which China will make up over 20 billion. Among all types of terminals, smart phones will contribute most of the traffic and IoT terminals will contribute less, even though the number of devices is much larger.

Towards year 2020 and beyond, the typical trends could be summarized as follows.

(i) Explosive Growth of Data Traffic. There will be explosive growth in traffic; the global data traffic will increase by more than 200 times from 2010 to 2020 and about 20000 times from 2010 to 2030 .

(ii) Great Increase of Devices in Connection. While smart phones are expected to remain as the main personal devices, the number of other kinds of devices, including wearable devices and MTC devices, will continuously increase.

(iii) Continuous Emergence of New Services. Different kinds of services, for example, services from enterprises, from vertical industries, and from Internet companies, will be exploited.

To meet the service and market demand towards year 2020 and beyond, the IMT-2020 is targeted to be deployed by year 2020 (5G) and meet new and unprecedented demands beyond the capability of previous generation systems. 5G will break through the limitation of time and space to enable an immersive and interactive user experience. $5 \mathrm{G}$ will also shorten the distance between human and things and implement seamless integration to achieve an easy and smart 

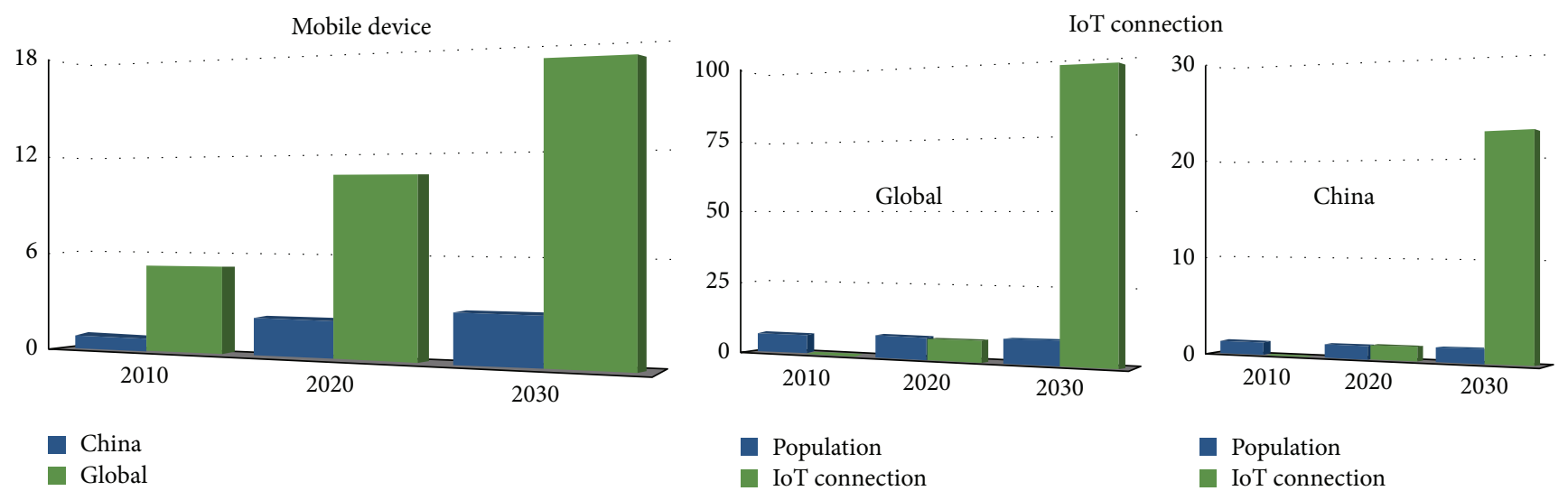

FIGURE 4: 2010-2030 growth of mobile device and IoT connection (unit: billion) [10].

interconnection between people and all things. 5G will provide users with fiber-like access data rate and "zero" latency user experience. $5 \mathrm{G}$ will be capable of connecting 100 billion devices. 5G will be able to deliver a consistent experience across a variety of scenarios including the cases of ultra-high traffic volume density, ultra-high connection density, and ultra-high mobility. $5 \mathrm{G}$ will also be able to provide intelligent optimization based on services and users awareness and will improve energy and cost efficiency by over a hundred of times, enabling us all to realize the vision of $5 \mathrm{G}$, "information a finger away, everything in touch."

\section{Services, Scenarios, and Performance Challenges}

As described in Figure 2, the future mobile communication system will penetrate to every corner of the society and bring us the immersive user experience. To derive the requirements for the $5 \mathrm{G}$ system, the typical service and user demand will be the mandatory requirements for the $5 \mathrm{G}$, while the typical deployment scenarios will also bring some bottlenecks to be solved by $5 \mathrm{G}$ system, especially from the domestic and commercial application perspective, for example, the cost and efficiency. This section identifies the service and user demands beyond year 2020 according to the possible popular services and user behavior in $5 \mathrm{G}$ era and derives the specific requirements of $5 \mathrm{G}$ system from the typical usage scenarios perspective.

3.1. Services and User Demands. Mobile Internet is aiming at people-oriented communications with a focus on user experience. Towards the year 2020 and beyond, the increasing popularity of ultra-high-definition (UHD) and 3D and video immersion will significantly drive up the data rates. For example, with a hundredfold compression, the transmission of $8 \mathrm{~K}(3 \mathrm{D})$ video will require a transmission rate close to 1 Gbps. Services, such as augmented reality, desktop cloud, and online gaming, will not only pose a challenge for uplink and downlink data transmission rates but also generate stringent demand for the so-called imperceptible latency. In the future, vast amounts of individuals and office data will be stored in the cloud. Such massive data activity will require transmission rates to be comparable to optical fiber communications, which will lead to enormous traffic challenges for mobile communications networks particularly in hotspot areas. Over-the-top (OTT) services, such as social networking, will be counted among leading applications going forward, and the associated frequently occurring small packets will devour signaling resources. At the same time, consumers will continue to demand better experiences on mobile communications wherever they are. A consistent service experience is expected in all kinds of scenarios, including ultra-dense scenarios such as stadiums, open-air gatherings and concerts, and high-speed moving scenarios such as highspeed trains, vehicles, and subways.

IoT is focused on communications between things and between things and people, involving not only individual users, but also a large number of various vertical industrial customers. The IoT services types and relevant requirements of IoT services are very diverse. For services such as smart home, smart grid, environmental monitoring, smart agriculture, and smart metering, the network will be required to support a massive amount of device connections and frequently occurring small data packets. Services like video surveillance and mobile health will have strict requirements on transmission rates, while services such as IoV and industrial control will demand millisecond-level latency and nearly $100 \%$ reliability. In addition, many IoT devices may be deployed in remote areas such as mountains, forests, and bodies of waters or in areas where transmission losses can be a problem, such as indoor corners, basements, and tunnels. Therefore, the coverage of mobile communications network needs to be further enhanced. In order to penetrate into more IoT services, 5G should be more flexible and more scalable, to support massive device connections and meet diverse user requirements.

Users expect better, yet more cost-effective, services and experiences with mobile Internet and the IoT. In addition to satisfying cost and experience demands, $5 \mathrm{G}$ will also need to meet extremely high security requirements, particularly for services such as e-banking, security monitoring, safe driving, and mobile health. $5 \mathrm{G}$ will also be able to support lower power 


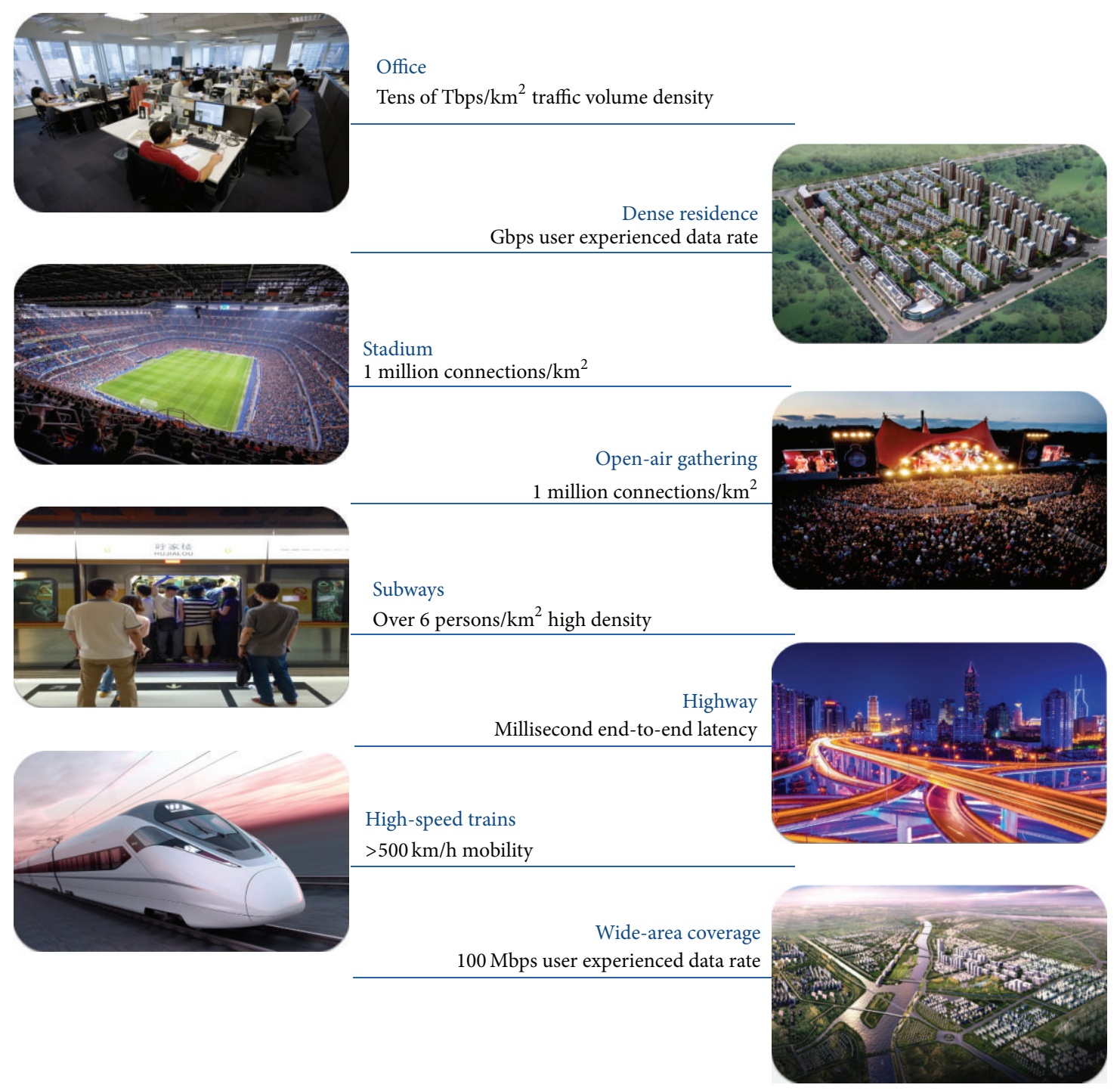

FIGURE 5: Challenging scenarios and performance.

consumption to build a greener mobile communication network and to enable much longer terminal battery life, especially for some IoT devices.

3.2. Scenarios and Performance Challenges. $5 \mathrm{G}$ will touch many aspects of life in the future, such as residence, office, leisure, and transportation. The 5G scenarios include at least dense residential areas, office towers, stadiums, openair gatherings, subways, fast ways, high-speed railways, and wide-area coverage. These scenarios, which are characterized by high traffic volume density or high connection density or high mobility, may be quite challenging for $5 \mathrm{G}$ as described in Figure 5.

Some typical services, such as augmented reality, virtual reality, ultra-high-definition videos, cloud storage, Internet of Vehicles, smart home, and OTT services, will occur in these scenarios. The performance requirements for $5 \mathrm{G}$ are derived for each scenario, according to the predicted distribution of users, percentage of different services, and service requirements such as data rate and latency. The key performance indicators for $5 \mathrm{G}$ are listed in Table 1, which include user experienced data rate, connection density, end-to-end delay, traffic volume density, mobility, and peak date rate.

\section{Sustainability and Efficiency Requirements}

To provide the immersive services to the users in an affordable way, the cost and efficiency of $5 \mathrm{G}$ system need to be considered when it is deployed and maintained after the commercial deployment. As forecasted in Figure 3, the mobile data traffic by year 2020 could grow by more than 1000 times, while it is not possible for the user to pay for the mobile data approximately to the data volume. To achieve this, the high cost efficiency of the network will be a key to make a successful $5 \mathrm{G}$. To reduce the cost per bit of $5 \mathrm{G}$, the spectrum efficiency of $5 \mathrm{G}$, the flexibility to adaptation to differentiated 
TABLE 1: $5 \mathrm{G}$ performance indicators.

\begin{tabular}{ll}
\hline Performance indicators & Definition \\
\hline User experienced data rate $(\mathrm{bps})$ & The minimum achievable data rate for a user in real network environment \\
Connection density $\left(/ \mathrm{km}^{2}\right)$ & $\begin{array}{l}\text { The total number of connected devices per unit area } \\
\text { The duration between the transmission of a data packet from the source node and the successful } \\
\text { End-to-end latency }(\mathrm{ms})\end{array}$ \\
$\begin{array}{l}\text { reception at destination node } \\
\text { Traffic volume density }\left(\mathrm{bps} / \mathrm{km}^{2}\right)\end{array}$ & $\begin{array}{l}\text { The total data rate of all users per unit area } \\
\text { Mobility }(\mathrm{km} / \mathrm{h})\end{array}$ \\
Peak date rate $(\mathrm{bps})$ & The maximum achievable data rate per user \\
\hline
\end{tabular}

usage scenarios and services, and simplified network optimization and maintenance are highly expected from $5 \mathrm{G}$.

4.1. Sustainability. Several problems are anticipated if today's networks are used to handle the explosive development of mobile Internet and IoT as follows.

(i) The energy efficiency level, overall cost per bit, and complexity of network deployment and maintenance cannot effectively handle the 1000-time traffic growth and a massive amount of connected devices in the future next decade.

(ii) Coexistence of multiple radio access technologies (RAT) causes increased complexity and degraded user experience.

(iii) Existing networks cannot realize accurate monitoring of network resources and effective awareness of services, and therefore they cannot intelligently fulfill the diversified requirements of future users and services.

(iv) Widely distributed and fragmented spectrum will cause interference and coexistence complexity.

To solve these problems, 5G should have the following capabilities to achieve sustainability. In terms of network construction and deployment, $5 \mathrm{G}$ needs to

(i) provide higher network capacity and better coverage, while decreasing the complexity and cost of network deployment, especially the deployment of ultra-dense networks;

(ii) have a flexible and scalable architecture to adapt to the diverse needs of users and services;

(iii) make flexible and efficient use of various spectrum resources, including paired and unpaired spectrum, refarmed spectrum and new spectrum, lowfrequency and high-frequency bands, and licensed and unlicensed bands;

(iv) have stronger device-connection capabilities to deal with the access requirements of huge amounts of IoT devices.

to

In terms of operation and maintenance (O\&M), 5G needs

(i) improve network energy efficiency and the O\&M cost per bit to cope with data traffic growth and the diverse needs of various services and applications;
TABLE 2: 5G key efficiency indicators.

\begin{tabular}{ll}
\hline Efficiency indicators & Definition \\
\hline $\begin{array}{l}\text { Spectrum efficiency } \\
\text { (bps/Hz/cell or } \\
\text { bps } / \mathrm{Hz} / \mathrm{km}^{2} \text { ) }\end{array}$ & $\begin{array}{l}\text { The data throughput per unit of spectrum } \\
\text { resource per cell (or per unit area) }\end{array}$ \\
$\begin{array}{l}\text { Energy efficiency } \\
\text { (bit/J) }\end{array}$ & $\begin{array}{l}\text { The number of bits that can be } \\
\text { transmitted per joule of energy } \\
\text { Cost efficiency (bit/Y) }\end{array}$ \\
$\begin{array}{l}\text { The number of bits that can be } \\
\text { transmitted per unit cost }\end{array}$ \\
\hline
\end{tabular}

(ii) reduce the complexity caused by the coexistence of multiple radio access technologies, network upgrades, and the introduction of new features and functions, to improve users' experience;

(iii) make intelligent optimization based on awareness of users behavior and services contents;

(iv) provide a variety of network security solutions to meet the needs of all types of devices and services of mobile Internet and IoT.

4.2. Efficiency Requirements. Spectrum utilization, energy consumption, and cost are the three key factors which must be addressed in sustainable mobile communication networks. In order to achieve sustainability, 5G needs to make significant improvements in the following aspects:

(i) Spectrum efficiency: 3 5 times.

(ii) Energy efficiency: 100+ times.

(iii) Cost efficiency: $100+$ times.

The definitions of these efficiency indicators are listed in Table 2.

\section{Key Capabilities of 5 G}

5G systems must dramatically outperform previous generation systems. $5 \mathrm{G}$ should support the following:

(i) User experienced data rate: 0.1 1 Gbps.

(ii) Connection density: 1 million connections per square kilometer.

(iii) End-to-end latency: millisecond level.

(iv) Traffic volume density: tens of Gbps per square kilometer. 


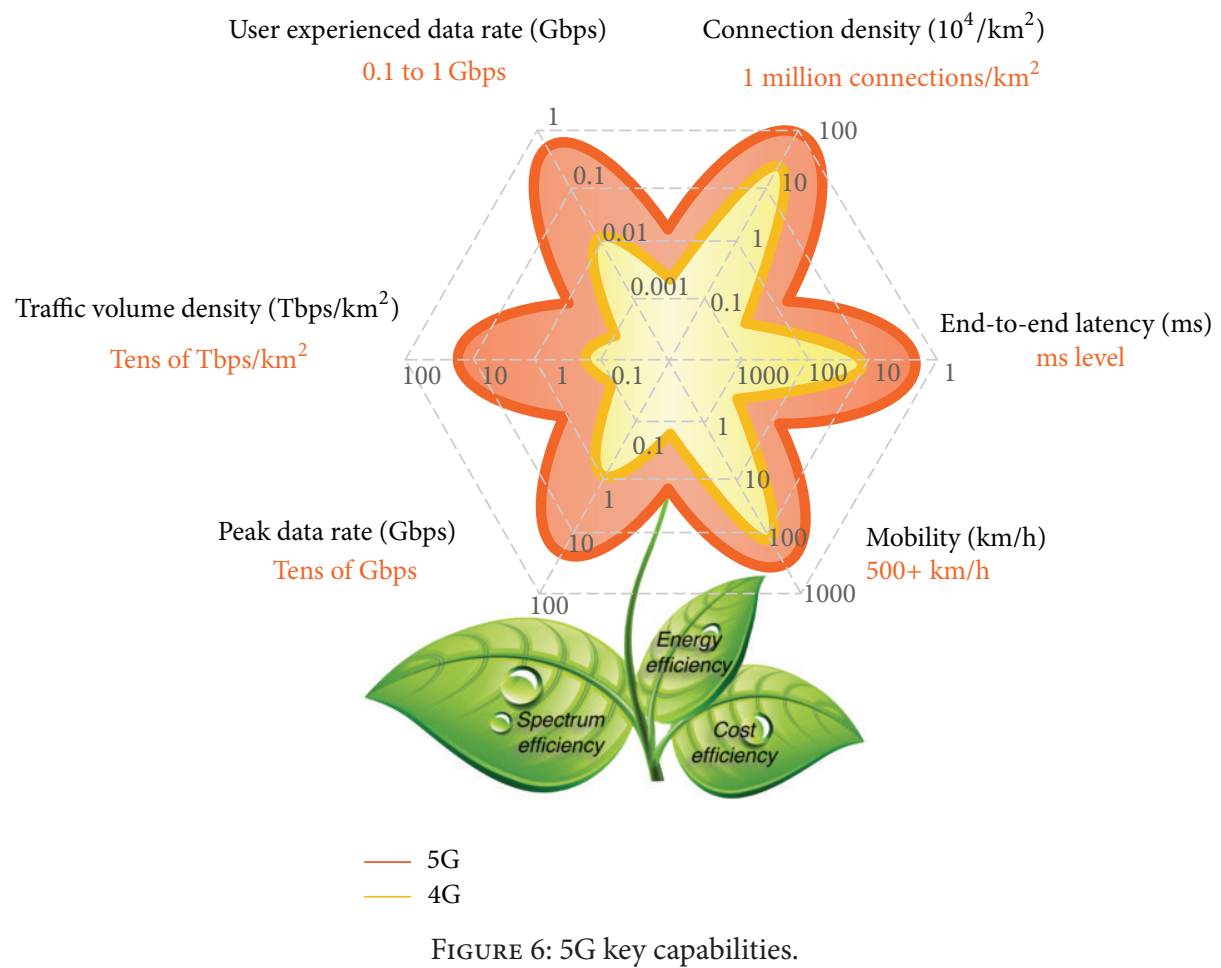

(v) Mobility: higher than $500 \mathrm{Km}$ per hour.

(vi) Peak data rate: tens of Gbps.

Among these requirements, user experienced data rate, connection density, and end-to-end latency are the three most fundamental ones. Meanwhile, $5 \mathrm{G}$ needs to significantly improve the efficiency of network deployment and operations. Compared with 4G, 5G should have 3 to 5 times' improvement on spectrum efficiency and more than 100 times' improvement on energy and cost efficiency.

The performance requirements and efficiency requirements define the key capabilities of 5G, which can be illustrated as a "blooming flower" in Figure 6. The petals and leaves rely on each other. The petals represent the six key capabilities in terms of performance and can fulfill the diverse requirements of future services and scenarios. The leaves represent the three key capabilities in terms of efficiency and can guarantee the sustainable development of $5 \mathrm{G}$. The top of each petal means the maximum value of the corresponding capability.

\section{Candidate Technologies and Spectrum}

To achieve the above $5 \mathrm{G}$ requirement objectives, there are some emerging technologies proposed by many organizations and companies including the following:

(i) Novel multiple access, such as SCMA (Sparse Code Multiple Access), MUSA (Multiuser Shared Access), PDMA (Pattern Division Multiple Access), and RSMA (Resource Spread Multiple Access) (this kind of technologies can increase the spectrum efficiency, user experienced data rate, system capacity, and connection density).

(ii) New waveforms, like filtered-OFDM, UFMC (Universal Filtered Multicarrier), window-OFDM, and so forth, to maximize the spectrum utilization.

(iii) Massive MIMO technologies to increase the spectrum efficiency, user experienced data rate, and system capacity.

(iv) New channel coding such as polar code and LDPC (Low Density Parity Check Code), to increase the spectrum efficiency.

(v) Software defined air interface and end-to-end network slicing to increase the system flexibility to support all kinds of services and to enhance the overall network cost efficiency.

There are three solutions to satisfy the increasing spectrum demands for $5 \mathrm{G}$ services. Identification of more spectra both below and above $6 \mathrm{GHz}$ for IMT is the solution with highest priority. Bands below $6 \mathrm{GHz}$ are the core spectrum bands used for IMT, with $\mathrm{C}$ band being a key band in the near future. The second one is to refarm spectrum used by legacy systems. The third one is to promote new technologies to share spectrum of the other radio services.

\section{Conclusions}

$5 \mathrm{G}$ will be able to sustainably satisfy the requirement of the 1000-time traffic growth. $5 \mathrm{G}$ will provide users with fiber-like access data rate and "zero" latency user experience. $5 \mathrm{G}$ will be capable of connecting 100 billion devices. 5G will be able 
to deliver a consistent experience across a variety of scenarios including the cases of ultra-high traffic volume density, ultrahigh connection density, and ultra-high mobility. 5G will also be able to provide intelligent optimization based on services and users awareness and will improve energy and cost efficiency by over a hundred of times, enabling us all to realize the vision of 5G, "information a finger away, everything in touch.”

\section{Competing Interests}

The authors declare that they have no competing interests.

\section{Acknowledgments}

This work is supported by the National Science and Technology Major Project under Grant no. 2015ZX03002005-002.

\section{References}

[1] METIS Report, "Scenarios, requirements and KPIs for 5G mobile and wireless system," 2013.

[2] W. Roh, J.-Y. Seol, J. Park et al., "Millimeter-wave beamforming as an enabling technology for $5 \mathrm{G}$ cellular communications: theoretical feasibility and prototype results," IEEE Communications Magazine, vol. 52, no. 2, pp. 106-113, 2014.

[3] E. G. Larsson, O. Edfors, F. Tufvesson, and T. L. Marzetta, "Massive MIMO for next generation wireless systems," IEEE Communications Magazine, vol. 52, no. 2, pp. 186-195, 2014.

[4] H. Nikopour, E. Yi, A. Bayesteh et al., "SCMA for downlink multiple access of 5G wireless networks," in Proceedings of the IEEE Global Communications Conference (GLOBECOM '14), pp. 3940-3945, Austin, Tex, USA, December 2014.

[5] B. Li, H. Shen, D. Tse, and W. Tong, "Low-latency polar codes via hybrid decoding," in Proceedings of the 8th International Symposium on Turbo Codes and Iterative Information Processing (ISTC '14), pp. 223-227, IEEE, Bremen, Germany, August 2014.

[6] C. Zhang and K. Parhi, "Low-latency sequential and overlapped architectures for successive cancellation polar decoder," IEEE Transactions on Signal Processing, vol. 61, no. 10, pp. 2429-2441, 2013.

[7] B. Yuan and K. K. Parhi, "Low-latency successive-cancellation polar decoder architectures using 2-bit decoding," IEEE Transactions on Circuits and Systems I: Regular Papers, vol. 61, no. 4, pp. 1241-1254, 2014.

[8] ITU, "Workplan, timeline, process and deliverables for the future development of IMT," ITU-R WP5D, 2015.

[9] "Summary of 3GPP RAN 5G workshop," 3GPP RWS-150073, ftp://ftp.3gpp.org/workshop/2015-09-17_18_RAN_5G/Docs/RWS150073.zip.

[10] IMT-2020 (5G) Promotion Group, “5G vision and requirements," http://www.imt-2020.cn. 


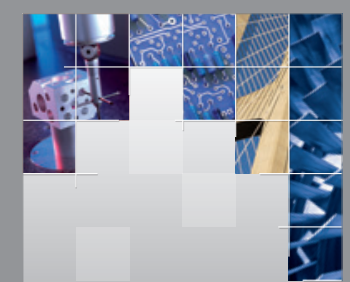

\section{Enfincering}
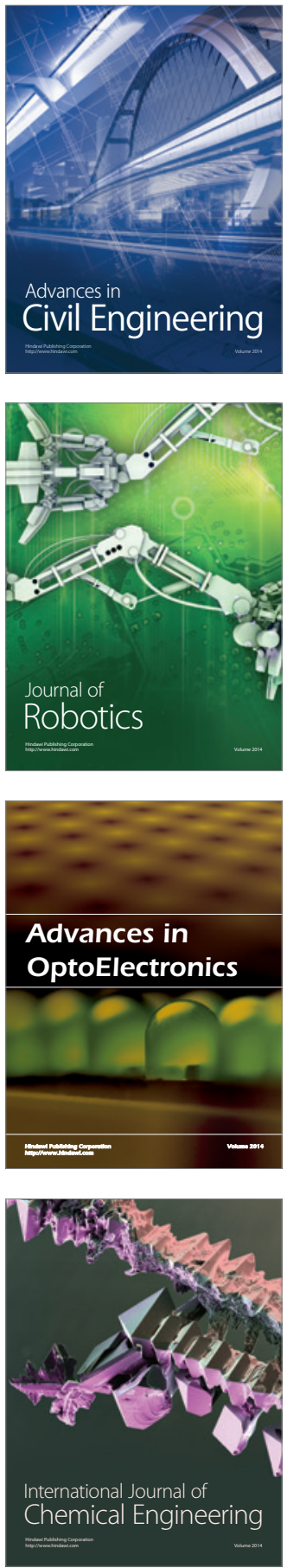

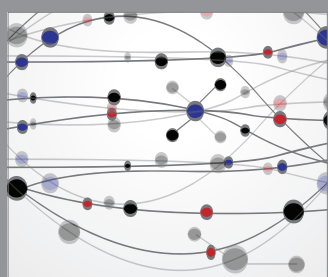

The Scientific World Journal

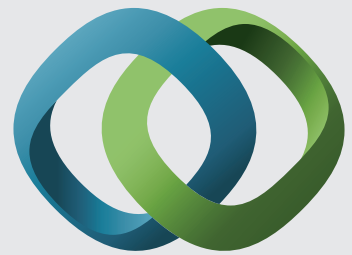

\section{Hindawi}

Submit your manuscripts at

http://www.hindawi.com
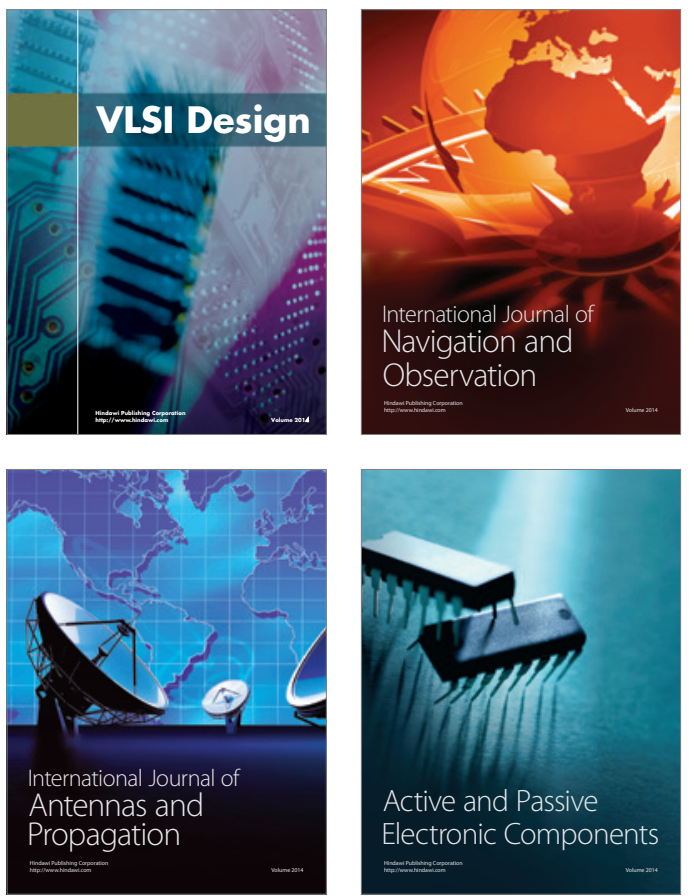
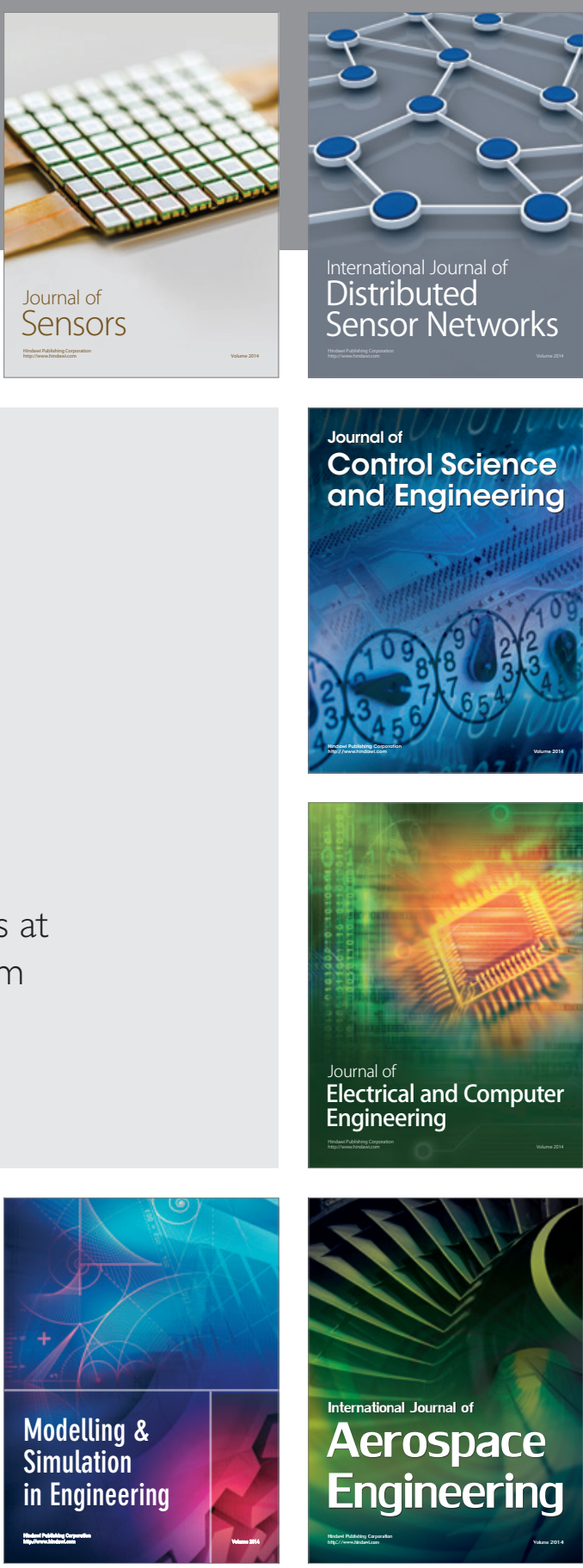

International Journal of

Distributed

Sensor Networks

Journal of

Control Science

and Engineering
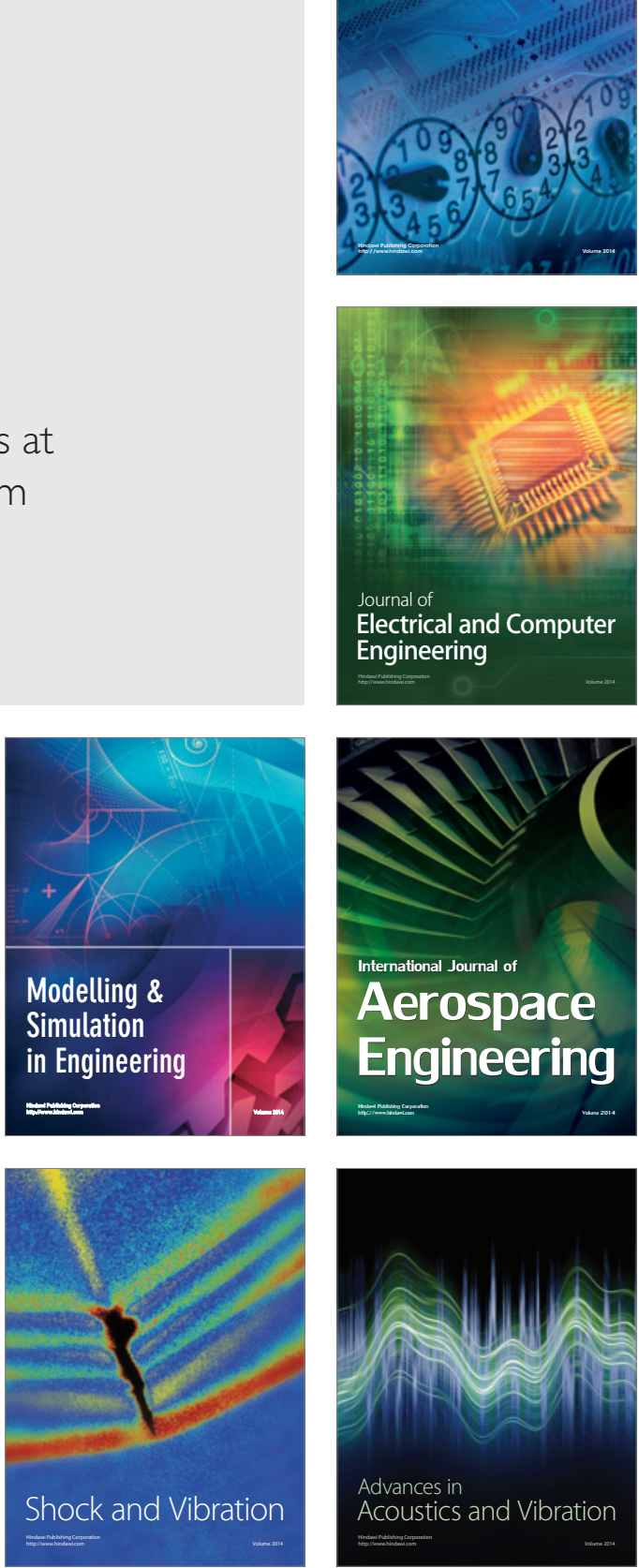